\title{
Expression Of Inflammatory Markers In The Muscles Of Patients With Idiopathic Inflammatory Myopathy According To The Presence Of Interstitial Lung Disease
}

\section{Jung Sun Lee}

Seoul Veterans Hospital

\section{Byeongzu Ghang}

Jeju National University School of Medicine

Wonho Choi

University of Ulsan College of Medicine

\section{Seokchan Hong}

University of Ulsan College of Medicine

\section{Yong-Gil Kim}

University of Ulsan College of Medicine

Chang-Keun Lee

University of Ulsan College of Medicine

\section{Soo Jeong Nam}

University of Ulsan College of Medicine

Bin Yoo ( $\sim$ byoo@amc.seoul.kr)

University of Ulsan College of Medicine

\section{Research Article}

Keywords: Myositis, Polymyositis, Dermatomyositis, interstitial lung disease, HLA-DR Antigens

Posted Date: February 15th, 2022

DOI: https://doi.org/10.21203/rs.3.rs-1271453/v2

License: (c) (i) This work is licensed under a Creative Commons Attribution 4.0 International License. Read Full License 


\section{Abstract}

Objective. Several studies have identified factors associated with the development of interstitial lung disease (ILD) in patients with idiopathic inflammatory myopathies (IIMs). However, few have assessed the association between ILD and muscle biopsy findings including inflammatory marker expressions analyzed using immunohistochemistry (IHC). We investigated the muscle biopsy findings that were associated with the presence of ILD.

Methods. Muscle biopsies from patients who were newly diagnosed with IIMs between 2000 and 2017 were reviewed. ILD was diagnosed based on high-resolution computed tomography findings at the time of diagnosis of IIMs. IHC staining was performed for CD3, CD4, CD8, CD20, CD68, CD163, MX1, MHC class I, and HLA-DR. The factors associated with the presence of ILD were evaluated by logistic regression analysis.

Results. Of the 129 patients with IIM, 49 (38\%) had ILD. In muscle biopsy findings, CD4 expression (47\% vs. $29 \%, P=0.036), \mathrm{MX} 1$ expression on immune cell $(29 \%$ vs. $13 \%, P=0.023)$, and expression of MHC class I ( $67 \%$ vs. $41 \%, P=0.004)$ and HLA-DR ( $37 \%$ vs. $11 \%, P=0.001)$ on myofiber were more common in patients with ILD than those without. In logistic regression analysis, HLA-DR expression on myofiber was significantly associated with the risk of ILD $(\mathrm{OR}, 2.39 ; 95 \% \mathrm{Cl}, 1.24-4.90, P=0.012)$ after adjusting for pathologic findings, clinical features, and autoantibodies.

Conclusion. Expression of HLA-DR on myofibers was associated with the presence of ILD in patients with IIM.

\section{Introduction}

Idiopathic inflammatory myopathies (IIMs) are systemic autoimmune disorders characterized by proximal muscle weakness and extra-muscular manifestation [1]. The lung is the most common extramuscular target next to the skin, and the frequency of interstitial lung disease (ILD) is as high as $20-40 \%$ in patients with IIM [2,3]. Because ILD is associated with increased mortality in patients with IIM [4], understanding and predicting the development of ILD in patients with IIM is an important issue. The known factors associated with the risk of ILD are malignancy and autoantibodies including anti-tRNAsynthetase, anti-PM-Scl, anti-Ro-52, and anti-melanoma differentiation-associated protein-5 (MDA5) autoantibodies [5-7]. However, the association between the development of ILD and various factors in the muscle, the most important site of involvement in IIMs, is not well-known.

The characteristic histopathologic features of IIMs include the presence of mononuclear inflammatory cell infiltrates in muscle tissue, necrotic muscle fibers, and regenerating muscle fibers [8-10]. Recent studies have reported that various inflammatory markers including major histocompatibility complex (MHC) class, T cell, B cell, macrophage, and type-1 interferon were associated with IIMs. [11-19] Because IIMs with ILD have inflammation in both the muscles and the lungs, the histopathologic features and 
expression of various inflammatory markers in muscle tissue could be important for understanding the pathogenesis of ILD in patients with IIM.

However, there were no studies that evaluated the association between the presence of ILD in patients with IIM and muscle biopsy findings including inflammatory markers using IHC analysis. Thus, we investigated the factors associated with the presence of ILD including various inflammatory markers in muscle biopsy specimens using IHC analysis.

\section{Materials And Methods 2.1 Study population}

In this retrospective cohort study, we reviewed the electronic medical records of patients who were newly diagnosed with IIMs and underwent muscle biopsy between January 2000 and April 2017 at Asan Medical Center, a tertiary referral hospital in Seoul, South Korea. IIMs were diagnosed based on the fulfillment of the 2017 European League Against Rheumatism/American College of Rheumatology classification criteria for adult and juvenile IIM [20]. At the time of diagnosis of IIMs, chest computed tomography (CT) was performed for cancer screening and the presence of ILD was investigated. Patients under 18 years of age at diagnosis and those diagnosed with inclusion body myositis were excluded.

We gathered information on the following data that were acquired at diagnosis: demographic variables (e.g., age, sex); presence of ILD and dysphagia according to high-resolution CT and videofluoroscopic swallowing study, respectively; and laboratory findings (e.g., myoglobin, creatine kinase, aldolase, lactate dehydrogenase, autoantibody profiles [i.e., anti-Ro, anti-Jo-1], C-reactive protein, erythrocyte sedimentation rate).

\subsection{Histopathologic and immunohistochemistry analysis}

Histopathologic features of muscle biopsy specimens were reviewed by a neuropathologist (SJ Nam), who re-assessed the typical histological findings of inflammatory myositis (e.g., fiber necrosis, fiber size variation [atrophic fibers], internal nuclei, moth-eaten fibers, core-like area and fiber) in each slide. The severity of histopathologic features was graded from 0 to 3 ( $0=$ none; $1=$ mild; $2=$ moderate; $3=$ severe $)$.

Whole sections of representative formalin-fixed paraffin-embedded tissue blocks of muscle specimens were used for further IHC analysis. IHC analysis was conducted using CD3, CD4, and CD8 as T cell markers, CD20 as a B cell marker, CD68 as a macrophage marker, MX1 as a systemic inflammatory marker associated with type 1 interferon, HLA-ABC as an MHC class I marker, and HLA-DR as an MHC class II marker.

The following antibodies were used: CD3 (1:100, Rabbit monoclonal, clone POLY, catalog No.A0452, DAKO, Glostrup, Denmark), CD4 (1:16, Rabbit monoclonal, clone SP35, catalog No.790-4423, Ventana Medical Systems, Tucson, AZ, USA), CD8 (1:400, Mouse monoclonal, clone C8/144B, catalog No.M7103, Cell Marque, Rocklin, CA, USA), CD20 (1:400, Mouse monoclonal, clone L26, catalog No.M0755, DAKO, 
Glostrup, Denmark), CD68 (1:2000, Mouse monoclonal, clone KP1, catalog No.M0814, DAKO, Glostrup, Denmark), CD163 (1:400, Mouse monoclonal, clone MRQ-26, catalog No.163M-16, Cell Marque, Rocklin, CA, USA), MX1 (1:500, Rabbit polyclonal, clone N2C2, catalog No. GTX110256, GeneTex, Irvine, CA, USA), MHC Class I (1:10000, clone EMR8-5, catalog No. ab70328, Abcam, Cambridge, UK), HLA-DR (1:5000, clone TAL 1B5, catalog No. ab20181, Abcam, Cambridge, UK). IHC staining was performed using a BenchMark XT Autostainer (Ventana Medical Systems, Tucson, AZ, USA). The intensity of IHC markers was graded from 0 to 3 ( 0 = none; 1 = weak; 2 = moderate; $3=$ strong $)$, and grade 2 and 3 were considered as positive results.

\subsection{Statistical analysis}

Categorical variables are presented as $\mathrm{n}(\%)$ and continuous variables are presented as median (interquartile range) or mean \pm standard deviation. Between-group differences were assessed using Fisher's exact test or $\chi^{2}$ test for categorical variables and Mann-Whitney $U$ test or Student's $t$-test for continuous variables. Factors significantly associated with ILD were identified using logistic regression and their odds ratios (ORs) with 95\% confidence intervals (Cls) are presented. Variables that were considered clinically important for the treatment outcome and those with a $P$ value $<0.2$ in univariate analyses were included in the multivariable analysis; in all other analyses, $P$ values $<0.05$ were considered to denote statistical significance. In multivariable analysis, variables were further selected by backward stepwise regression and those with $P$ values $<0.05$ were retained in the final model. All statistical analyses were conducted using R v3.5.1 (The R Foundation for Statistical Computing, Vienna, Austria).

\section{Results}

\subsection{Comparison of clinical features according to the presence of ILD}

Table 1 shows the baseline clinical characteristics, laboratory findings, and pathologic findings according to the presence of ILD. Of the 129 patients with IIM, 49 had ILD (dermatomyositis, 55\% (27/66) vs. polymyositis, $45 \%(22 / 63), P=0.60)$. The most common ILD pattern was nonspecific interstitial pneumonia (53\%), followed by organizing pneumonia (37\%), usual interstitial pneumonia (6\%), and unclassified interstitial lung abnormalities (4\%). There were no significant differences in the clinical features between the ILD group and non-ILD group, but the proportions of patients positive for anti-Jo-1 ( $27 \%$ vs. $3 \%, P=0.001)$ and anti-Ro $(47 \%$ vs. $20 \%, P=0.007)$ were higher in the ILD group. In addition, CRP was higher in the ILD group as well. 
Table 1

Characteristics of idiopathic inflammatory myositis according to the presence of interstitial lung disease

$\begin{array}{ll}\text { ILD (+) } & \text { ILD }(-) \\ (n=49) & (n=80)\end{array}$

Diagnosis of IIMs

$27(55 \%)$

$39(49 \%)$

Dermatomyositis

$22(45 \%)$

$41(51 \%)$

Polymyositis

$54(42-60)$

$53(41-64.5)$

0.82

Age at diagnosis, years

$18(37 \%)$

$26(33 \%)$

0.76

Male sex

$10(20 \%)$

$23(29 \%)$

0.40

Heliotrope rash

$14(29 \%)$

$21(26 \%)$

0.93

Gottron's sign/papules

$7(14 \%)$

$22(28 \%)$

0.13

V or Shawl sign

$12(25 \%)$

27 (34\%)

0.36

Dysphagia

$43(88 \%)$

76 (95\%)

0.18

Proximal symmetric weakness

$35(80 \%)$

$69(91 \%)$

0.14

Myopathy on electromyography

Laboratory finding

Muscle enzyme

Creatine kinase, U/L

1505.0

2755

0.48

(502.0-53290)

(412.5-7404.0)

Myoglobin, $\mathrm{ng} / \mathrm{mL}$

559

752

0.63

(196.5-1714.0)

(223.0-2219.0)

Lactate dehydrogenase, $\mathrm{U} / \mathrm{L}$

627.0

587.0

0.88

(416.0-996.0)

(368.0-1197.0)

Autoantibodies

Anti-Jo $(n=108)$ *

$13(27 \%)$

$2(3.3 \%)$

0.001

Data are $\mathrm{n}(\%)$ or median (interquartile range).

ILD: interstitial lung disease, IIMs: idiopathic inflammatory myopathies, ESR: Erythrocyte sedimentation rate. CRP: C-reactive protein.

*: tested number. 


\begin{tabular}{|llll|}
\hline & $\begin{array}{l}\text { ILD (+) } \\
(\mathbf{n = 4 9 )}\end{array}$ & $\begin{array}{l}\text { ILD }(-) \\
(\mathbf{n}=\mathbf{8 0})\end{array}$ \\
\hline Anti-Ro $(\mathrm{n}=101)^{\star}$ & $21(47 \%)$ & $11(20 \%)$ & 0.007 \\
\hline ESR, $\mathrm{mm} / \mathrm{h}$ & $51(36-67)$ & $41(25.5-59.5)$ & 0.15 \\
\hline CRP, $\mathrm{mg} / \mathrm{dL}$ & $1.2(0.5-3.6)$ & $0.4(0.1-1.8)$ & 0.003 \\
\hline Pathologic finding & & & \\
\hline Fiber necrosis & $23(47 \%)$ & $45(56 \%)$ & 0.40 \\
\hline Fiber size variation atrophic fibers & $23(47 \%)$ & $76(95 \%)$ & 0.40 \\
\hline Internal nuclei & $26(53 \%)$ & $45(56 \%)$ & 0.86 \\
\hline Moth-eaten fibers & $1(2 \%)$ & $5(6 \%)$ & 0.41 \\
\hline Fiber splitting & $8(16 \%)$ & $21(26 \%)$ & 0.27 \\
\hline Perifascicularatrophy & $16(20 \%)$ & $7(14 \%)$ & 0.56 \\
\hline Endomyseal lymphocyte & $53(66 \%)$ & $33(67 \%)$ & 0.12 \\
\hline Perimyseal lymphocyte & $52(65 \%)$ & $39(80 \%)$ & \\
\hline Data are $n$ (\%) or median (interquartile range). & & \\
\hline ILD: interstitial lung disease, IIMs: idiopathic inflammatory myopathies, ESR: Erythrocyte & & \\
\hline sedimentation rate. CRP: C-reactive protein. & & & \\
\hline *: tested number. & & & \\
\hline 3 & & & \\
\hline
\end{tabular}

\subsection{Immunohistochemistry staining features according to the presence of ILD}

Table 2 shows the IHC staining features according to the presence of ILD. The ILD group had higher proportions of patients with CD4 expression (47\% vs. $29 \%, P=0.036$ ), Mx 1 expression on immune cells ( $29 \%$ vs. $13 \%, P=0.023)$, and expression of MHC class $1(68 \%$ vs. $41 \%, P=0.004)$ and HLA-DR on myofiber ( $37 \%$ vs. $11 \%, P=0.001$ ) than in the non-ILD group. Figure 1 shows the representative images of $\mathrm{CD} 4, \mathrm{Mx} 1, \mathrm{MHC}$ class I, and HLA-DR expression in muscle specimens. 
Table 2

IHC staining results according to the presence of interstitial lung disease ${ }^{a}$

\begin{tabular}{|c|c|c|c|}
\hline & $\begin{array}{l}\text { ILD (+) } \\
(n=49)\end{array}$ & $\begin{array}{l}\text { ILD }(-) \\
(n=80)\end{array}$ & $P$ \\
\hline \multicolumn{4}{|c|}{ Inflammatory cell expression } \\
\hline CD3 & $25(51 \%)$ & $27(34 \%)$ & 0.052 \\
\hline CD4 & $23(47 \%)$ & $23(29 \%)$ & 0.036 \\
\hline CD8 & $15(31 \%)$ & $14(18 \%)$ & 0.08 \\
\hline CD20 & $12(25 \%)$ & $10(13 \%)$ & 0.08 \\
\hline CD68 & $25(51 \%)$ & $30(38 \%)$ & 0.13 \\
\hline CD163 & $22(45 \%)$ & $32(40 \%)$ & 0.72 \\
\hline \multicolumn{4}{|c|}{ MX1 expression } \\
\hline Immune cell & $14(29 \%)$ & $10(13 \%)$ & 0.023 \\
\hline Myofiber & $10(20 \%)$ & $20(25 \%)$ & 0.55 \\
\hline Capillary & $34(69 \%)$ & $43(54 \%)$ & 0.08 \\
\hline \multicolumn{4}{|c|}{ Expression of MHC molecules on myofiber } \\
\hline MHC class 1 & $33(67 \%)$ & $33(41 \%)$ & 0.004 \\
\hline HLA-DR & $18(37 \%)$ & $9(11 \%)$ & 0.001 \\
\hline \multicolumn{4}{|c|}{ Data are $\mathrm{n}(\%)$. } \\
\hline \multicolumn{4}{|c|}{ a Moderate-to-strong intensities of staining were considered as positive results. } \\
\hline IHC: immunol & Complex & human le & te antic \\
\hline
\end{tabular}

\subsection{Factors associated with ILD in idiopathic inflammatory myositis patients}

Logistic regression analysis was performed to evaluate the factors associated with the presence of ILD in patients with IIM. In univariate analysis (Table 3), the titers of anti-Jo-1 and anti-Ro were significantly associated with the presence of ILD. In IHC staining, CD3 or CD4 expression, Mx1 expression on immune cell, and expression of MHC class I or HLA-DR on myofiber were associated with the presence of ILD. In multivariate analysis (Table 4), we created 3 models that were adjusted for IHC staining (model 1), laboratory finding, pathologic finding, and IHC staining (model 2), and clinical characteristics, laboratory finding, pathologic finding, and IHC staining (model 3). HLA-DR expression on myofiber was significantly associated with the presence of ILD in model $1(\mathrm{OR}, 1.97 ; 95 \% \mathrm{Cl}, 1.16-3.49 ; P=0.015)$ and model 3 (OR, 
2.39; $95 \% \mathrm{Cl}, 1.24-4.90 ; P=0.012)$. Increases in anti-Jo-1 titer were associated with the presence of ILD in model $2(\mathrm{OR}, 1.02 ; 95 \% \mathrm{Cl}, 1.00-1.05 ; P=0.058)$ and model $3(\mathrm{OR}, 1.02 ; 95 \% \mathrm{Cl}, 1.00-1.05 ; P=0.074)$, albeit without statistical significance. 
Table 3

Factors associated with the presence of interstitial lung disease in univariate analysis

\begin{tabular}{|c|c|c|c|}
\hline & OR & $95 \% \mathrm{Cl}$ & $P$ \\
\hline \multicolumn{4}{|l|}{ Clinical characteristics } \\
\hline Age at diagnosis & 1.01 & $0.98-1.03$ & 0.65 \\
\hline Male sex & 1.21 & $0.57-2.54$ & 0.62 \\
\hline Heliotrope rash & 0.64 & $0.26-1.45$ & 0.29 \\
\hline Gottron's sign/papules & 1.12 & $0.50-2.48$ & 0.77 \\
\hline V or shawl sign & 0.44 & $0.16-1.08$ & 0.09 \\
\hline Dysphagia & 0.64 & $0.28-1.39$ & 0.27 \\
\hline Proximal symmetric weakness & 0.38 & $0.09-1.39$ & 0.15 \\
\hline Myopathy on electromyography & 0.39 & $0.13-1.14$ & 0.09 \\
\hline Myalgia & 1.56 & $0.75-3.22$ & 0.23 \\
\hline \multicolumn{4}{|l|}{ Laboratory finding } \\
\hline Creatine kinase & 1.00 & $1.00-1.00$ & 0.29 \\
\hline ESR & 1.01 & $1.00-1.02$ & 0.21 \\
\hline CRP & 0.99 & $0.89-1.09$ & 0.86 \\
\hline Anti-Ro titer & 1.01 & $1.00-1.01$ & 0.016 \\
\hline Anti-Jo-1 titer & 1.03 & $1.00-1.06$ & 0.023 \\
\hline \multicolumn{4}{|l|}{ Pathologic finding ${ }^{\text {a }}$} \\
\hline Fiber necrosis & 1.09 & $0.66-1.80$ & 0.73 \\
\hline Fiber size variation atrophic fibers & 0.68 & $0.40-1.15$ & 0.16 \\
\hline Internal nuclei & 1.25 & $0.78-1.99$ & 0.35 \\
\hline Moth-eaten fibers & 0.31 & $0.02-2.02$ & 0.30 \\
\hline Fiber splitting & 0.55 & $0.21-1.32$ & 0.19 \\
\hline Perifascicularatrophy & 0.67 & $0.24-1.71$ & 0.41 \\
\hline Endomyseal lymphocyte & 1.10 & $0.74-1.67$ & 0.63 \\
\hline \multicolumn{4}{|c|}{ 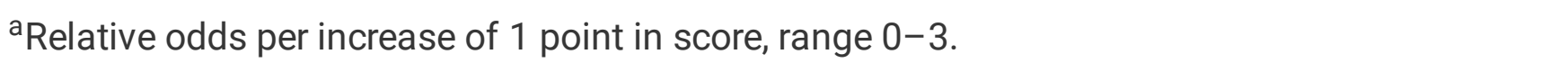 } \\
\hline
\end{tabular}




\begin{tabular}{|llll|}
\hline & OR & $95 \%$ Cl & $P$ \\
\hline Perimyseal lymphocyte & 1.20 & $0.92-1.58$ & 0.19 \\
\hline IHC staining ${ }^{\text {a }}$ & & & \\
\hline Inflammatory cell expression & & & \\
\hline CD3 & 1.67 & $1.03-2.77$ & 0.040 \\
\hline CD4 & 1.80 & $1.22-2.72$ & 0.004 \\
\hline CD8 & 1.43 & $0.92-2.25$ & 0.11 \\
\hline CD20 & 1.54 & $1.03-2.34$ & 0.039 \\
\hline CD68 & 1.58 & $0.93-2.72$ & 0.09 \\
\hline CD163 & 1.42 & $0.83-2.44$ & 0.20 \\
\hline MX1 expression & & & \\
\hline Immune cell & 1.89 & $1.16-3.16$ & 0.012 \\
\hline Myofiber & 0.84 & $0.56-1.22$ & 0.37 \\
\hline Capillary & 1.33 & $0.91-1.97$ & 0.14 \\
\hline Expression of MHC molecules on myofiber & & & \\
\hline MHC class 1 & 1.72 & $1.11-2.76$ & 0.019 \\
\hline HLA-DR & 2.18 & $1.40-3.53$ & $<0.001$ \\
\hline aRelative odds per increase of 1 point in score, range $0-3$. & & \\
\hline ESR: Erythrocyte sedimentation rate. CRP: C-reactive protein, IHC: immunohistochemistry, MHC: major & \\
\hline Histocompatibility Complex, HLA: human leukocyte antigen. & & \\
\hline
\end{tabular}


Table 4

Factors associated with the presence of interstitial lung disease in multivariable analysis

\begin{tabular}{|c|c|c|c|c|c|c|c|c|c|}
\hline \multirow[b]{2}{*}{ Factors } & \multicolumn{3}{|c|}{ Model 1* } & \multicolumn{3}{|c|}{ Model 2† } & \multicolumn{3}{|c|}{ Model $3^{\ddagger}$} \\
\hline & OR & $\begin{array}{l}95 \% \\
\mathrm{Cl}\end{array}$ & $P$ & OR & $\begin{array}{l}95 \% \\
\mathrm{Cl}\end{array}$ & $P$ & OR & $\begin{array}{l}95 \% \\
\mathrm{Cl}\end{array}$ & $P$ \\
\hline V or Shawl sign & & & & & & & 0.31 & $\begin{array}{l}0.08- \\
1.10\end{array}$ & 0.08 \\
\hline $\begin{array}{l}\text { Myopathy on } \\
\text { electromyography }\end{array}$ & & & & & & & 0.18 & $\begin{array}{l}0.02- \\
0.95\end{array}$ & 0.06 \\
\hline $\begin{array}{l}\text { Anti-Jo-1 titer (per } 1 \\
\mathrm{U} / \mathrm{mL} \text { increase) }\end{array}$ & & & & 1.02 & $\begin{array}{l}1.00- \\
1.05\end{array}$ & 0.06 & 1.02 & $\begin{array}{l}1.00- \\
1.05\end{array}$ & 0.07 \\
\hline CD4 expression ${ }^{a}$ & 1.76 & $\begin{array}{l}0.96- \\
3.31\end{array}$ & 0.07 & & & & & & \\
\hline CD8 expression ${ }^{a}$ & 0.56 & $\begin{array}{l}0.27- \\
1.13\end{array}$ & 0.11 & & & & 0.53 & $\begin{array}{l}0.24- \\
1.10\end{array}$ & 0.10 \\
\hline $\begin{array}{l}\text { MX1 expression on } \\
\text { immune cell }^{a}\end{array}$ & 1.51 & $\begin{array}{l}0.87- \\
2.67\end{array}$ & 0.15 & & & & & & \\
\hline $\begin{array}{l}\text { HLA-DR expression on } \\
\text { myofiber a }\end{array}$ & 1.97 & $\begin{array}{l}1.16- \\
3.49\end{array}$ & 0.015 & 1.62 & $\begin{array}{l}0.96- \\
2.85\end{array}$ & 0.08 & 2.39 & $\begin{array}{l}1.24- \\
4.90\end{array}$ & 0.012 \\
\hline \multicolumn{10}{|c|}{ *Model 1: adjusted for IHC staining } \\
\hline \multicolumn{10}{|c|}{ †Model 2: adjusted for laboratory finding + pathologic finding + IHC staining } \\
\hline ‡ Model 3: adjusted for & nical c & aracteri & $c+$ labo & atory $\mathrm{fi}$ & $\operatorname{ding}+\mathrm{p}$ & holog & findin & $+\mathrm{IHC} s$ & ining \\
\hline
\end{tabular}

\section{Discussion}

By analyzing the expression of inflammatory markers in the muscle specimens of 129 patients with IIM with or without ILD, we found multiple IHC markers (e.g., CD4, MX1 expression on immune cells, expression of MHC class I and HLA-DR on myofibers) that were differentially expressed according to the presence of ILD and significantly associated with ILD in logistic regression analysis. Specifically, regardless of the consideration for pathologic findings, clinical features, and autoantibodies, HLA-DR expression on myofibers consistently showed a significant association with the presence of ILD.

A study on anti-synthetase myopathy showed that the HLA-DR expression is correlated with $\mathrm{CD} 8^{+} \mathrm{T}$ cell infiltration, which suggests an involvement of the interferon-gamma pathway in the myofiber HLA-DR expression [21]. In IIM patients with ILD, T cell activation has an important role in lung damage [22], and a common pattern in the T cell receptor gene has been identified in both the lung and muscles [23]. Indeed, 
in lung biopsy specimens of patients with myositis-associated ILD, CD8 ${ }^{+} \mathrm{T}$ cells were increased and diffusely distributed in normal alveoli $[24,25]$. Furthermore, Jo- 1 antigen-reactive CD $4^{+} T$ cells were found in bronchoalveolar lavage fluid in patients with IIM/anti-synthetase syndrome and $\mathrm{CD} 4^{+} \mathrm{T}$ cells produce high levels of interferon-gamma [26]. In addition to these findings, we found that HLA-DR expression on myofiber is significantly associated with the presence of ILD in patients with IIM. As such, we speculate that HLA-DR expression on myofiber is associated with interferon-gamma-mediated CD $8^{+}$or $\mathrm{CD} 4^{+} \mathrm{T}$ cells that might contribute to the damages in muscle fibers and lungs in IIM patients with ILD. Although the mechanism of the association between myofiber HLA-DR expression and ILD could not be delineated in the present study, our findings provide additional information on the risk and pathogenesis of ILD in IIMs. Further studies are needed to examine the mechanism of the association between myofiber HLA-DR expression and ILD.

There are several studies on the association between antibodies and ILD in IIMs. Muscle-specific antibodies including anti-synthetase antibody and MDA-5 were shown to be associated with the presence of ILD and its prognosis.[27] Anti-synthetase antibodies are directed against tRNA-synthetases, whose proteins are not only an essential part of the translation apparatus but also have numerous cytoplasmic, nuclear, and extracellular functions, such as the triggering or silencing of inflammatory/immune responses, participation in lung development, and neuromuscular disorders.[28, 29] The most common anti-synthetase antibody is anti-Jo-1 antibody, which specifically recognizes the histidyl-tRNA-synthetase. Muscle tissues from patients with IIM showed a higher level of histidyl-tRNA-synthetase compared with those from healthy controls [30]. Histidine, which is incorporated into proteins by histidyl-tRNAsynthetase, is an essential proteinogenic amino acid with an imidazole functional group, which has antioxidant, antisecretory, and anti-inflammatory properties, and suppresses proinflammatory cytokine expression possibly through the NF-kB pathway.[31] Therefore, anti-Jo-1 antibody may have a significant role in autoimmune response.

Previous studies reported patients with anti-synthetase antibodies with ILD and no myositis. [32, 33] While the pathogenesis of anti-synthetase syndrome is suggested to be multifactorial, the lung was suggested as the site of first lesions. A previous study suggested that the lung might be the primary target of the autoimmune response via a novel antigen including Jo-1 or MDA, and the immune response might be directed against regenerating muscle cells [34]. Accordingly, in the present study, increases in anti-Jo-1 titer were associated with the presence of ILD, albeit without statistical significance. Anti-Jo-1 antibody may have an important role in the development of ILD in IIMs.

The present study has some limitations. First, because the study design was retrospective and performed in a single tertiary referral center, there were missing data regarding autoantibody test results and other autoantibodies such as MDA5, anti-tRNA-synthetase autoantibodies could not be evaluated. Second, because we could not evaluate the expression of inflammatory markers and their function in lung biopsy specimens, the mechanism of the association between HLA-DR expression and the presence of ILD could not be explained. However, our study has strength in that we analyzed the muscle specimens from more than 100 cases of IIMs and investigated the expression of various inflammatory markers using IHC 
staining. Considering the scarcity of studies that utilized IHC staining on muscle specimens of IIM, we believe that the significant associations of various IHC markers (e.g., CD3, CD4, CD20, MX1 expression on immune cells, expression of MHC class I and HLA-DR on myofibers) shown in logistic regression analyses would provide important clues to the pathogenesis of ILD in IIMs, which should be further investigated in targeted studies.

\section{Conclusions}

In patients with IIM, several inflammatory markers were differentially expressed in the muscle according to the presence of ILD. Especially, expression of HLA-DR in myofibers was consistently associated with ILD regardless of the consideration of pathologic findings, clinical features, and autoantibodies. Myofiber expression of HLA-DR could provide additional information on the risk of ILD in patients with IIM and a clue to the pathogenesis of ILD in IIMs.

\section{Abbreviations}

Cl: confidence intervals; CT: computed tomography; IHC: immunohistochemistry; IIM: idiopathic inflammatory myopathies; ILD: interstitial lung disease; MDA5: melanoma differentiation-associated protein-5; MHC: major histocompatibility complex; ORs: odds ratios

\section{Declarations}

\section{Ethics approval and consent to practice}

The study was approved by the Institutional Review Board of Asan Medical Center, Seoul, Korea (No. 2021-0881); the study was conducted according to the ethical principles of the Declaration of Helsinki. Patients' written informed consent was obtained.

\section{Consent for publication}

Not applicable.

\section{Availability of data and materials}

The datasets used and/or analysed during the current study are not publicly available due to patient privacy and confidentiality, but are available from the corresponding author upon reasonable request.

\section{Competing interests}

The authors declare that they have no conflicts of interest.

\section{Funding}


This work was supported by a research grant from the Jeju National University Hospital in 2018 (201820).

\section{Author Contributions}

Study concept and design: BG, SJN, BY. Data acquisition and analysis: JSL, WC, BG, SJN. Manuscript Writing: JSL, BG, SJN. Manuscript review: SH, YGK, CKL, BY. All authors contributed to discussions and read and approved the final manuscript.

\section{Acknowledgments}

We would like to thank Hwa Jung Kim from the Department of Clinical Epidemiology and Biostatistics. We thank Dr. Joon Seo Lim from the Scientific Publications Team at Asan Medical Center for his editorial assistance.

\section{References}

1. Malik A, Hayat G, Kalia JS, Guzman MA: Idiopathic Inflammatory Myopathies: Clinical Approach and Management. Front Neurol 2016, 7:64-64.

2. Marie I, Hachulla E, Chérin P, Dominique S, Hatron PY, Hellot MF, Devulder B, Herson S, Levesque H, Courtois H: Interstitial lung disease in polymyositis and dermatomyositis. Arthritis Rheum 2002, 47(6):614-622.

3. Kang EH, Lee EB, Shin $\mathrm{KC}$, Im CH, Chung DH, Han SK, Song YW: Interstitial lung disease in patients with polymyositis, dermatomyositis and amyopathic dermatomyositis. Rheumatology 2005 , 44(10):1282-1286.

4. Johnson C, Pinal-Fernandez I, Parikh R, Paik J, Albayda J, Mammen AL, Christopher-Stine L, Danoff S: Assessment of Mortality in Autoimmune Myositis With and Without Associated Interstitial Lung Disease. Lung 2016, 194(5):733-737.

5. Lega J-C, Reynaud Q, Belot A, Fabien N, Durieu I, Cottin V: Idiopathic inflammatory myopathies and the lung. European Respiratory Review 2015, 24(136):216-238.

6. Shappley C, Paik JJ, Saketkoo LA: Myositis-Related Interstitial Lung Diseases: Diagnostic Features, Treatment, and Complications. Curr Treatm Opt Rheumatol 2019, 5(1):56-83.

7. Zhang L, Wu G, Gao D, Liu G, Pan L, Ni L, Li Z, Wang Q: Factors Associated with Interstitial Lung Disease in Patients with Polymyositis and Dermatomyositis: A Systematic Review and MetaAnalysis. PLOS ONE 2016, 11(5):e0155381.

8. Dalakas MC: Polymyositis, dermatomyositis and inclusion-body myositis. N Engl J Med 1991, 325(21):1487-1498.

9. Plotz PH, Rider LG, Targoff IN, Raben N, O'Hanlon TP, Miller FW: NIH conference. Myositis: immunologic contributions to understanding cause, pathogenesis, and therapy. Ann Intern Med 1995, 122(9):715-724. 
10. Engel AG, Arahata K: Mononuclear cells in myopathies: quantitation of functionally distinct subsets, recognition of antigen-specific cell-mediated cytotoxicity in some diseases, and implications for the pathogenesis of the different inflammatory myopathies. Hum Pathol 1986, 17(7):704-721.

11. Huang K, Li QX, Bi FF, Duan HQ, Mastaglia F, Luo YB, Yang H: Comparative immunoprofiling of polymyositis and dermatomyositis muscles. Int J Clin Exp Pathol 2018, 11(8):3984-3993.

12. Choi J-H, Park Y-E, Kim S-I, Kim J-I, Lee C-H, Park K-H, Kim D-S: Differential immunohistological features of inflammatory myopathies and dysferlinopathy. J Korean Med Sci 2009, 24(6):10151023.

13. Soponkanaporn S, Deakin CT, Schutz PW, Marshall LR, Yasin SA, Johnson CM, Sag E, Tansley SL, McHugh NJ, Wedderburn LR et al: Expression of myxovirus-resistance protein A: a possible marker of muscle disease activity and autoantibody specificities in juvenile dermatomyositis. Neuropathol Appl Neurobiol 2019, 45(4):410-420.

14. Rodríguez Cruz PM, Luo YB, Miller J, Junckerstorff RC, Mastaglia FL, Fabian V: An analysis of the sensitivity and specificity of MHC-I and MHC-II immunohistochemical staining in muscle biopsies for the diagnosis of inflammatory myopathies. Neuromuscul Disord 2014, 24(12):1025-1035.

15. Dorph C, Englund P, Nennesmo I, Lundberg IE: Signs of inflammation in both symptomatic and asymptomatic muscles from patients with polymyositis and dermatomyositis. Annals of the rheumatic diseases 2006, 65(12):1565-1571.

16. Peng QL, Zhang YL, Shu XM, Yang HB, Zhang L, Chen F, Lu X, Wang GC: Elevated Serum Levels of Soluble CD163 in Polymyositis and Dermatomyositis: Associated with Macrophage Infiltration in Muscle Tissue. J Rheumatol 2015, 42(6):979-987.

17. Baechler EC, Bilgic $H$, Reed AM: Type I interferon pathway in adult and juvenile dermatomyositis. Arthritis Res Ther 2011, 13(6):249.

18. Liao AP, Salajegheh M, Nazareno R, Kagan JC, Jubin RG, Greenberg SA: Interferon $\boldsymbol{\beta}$ is associated with type 1 interferon-inducible gene expression in dermatomyositis. Ann Rheum Dis 2011, 70(5):831-836.

19. Kao L, Chung L, Fiorentino DF: Pathogenesis of dermatomyositis: role of cytokines and interferon. Curr Rheumatol Rep 2011, 13(3):225-232.

20. Lundberg IE, Tjärnlund A, Bottai M, Werth VP, Pilkington C, Visser M, Alfredsson L, Amato AA, Barohn RJ, Liang MH et al: 2017 European League Against Rheumatism/American College of Rheumatology classification criteria for adult and juvenile idiopathic inflammatory myopathies and their major subgroups. Ann Rheum Dis 2017, 76(12):1955-1964.

21. Aouizerate J, De Antonio M, Bassez G, Gherardi RK, Berenbaum F, Guillevin L, Berezne A, Valeyre D, Maisonobe T, Dubourg 0 et al: Myofiber HLA-DR expression is a distinctive biomarker for antisynthetase-associated myopathy. Acta Neuropathol Commun 2014, 2:154.

22. Saketkoo LA, Ascherman DP, Cottin V, Christopher-Stine L, Danoff SK, Oddis CV: Interstitial Lung Disease in Idiopathic Inflammatory Myopathy. Curr Rheumatol Rev 2010, 6(2):108-119. 
23. Englund P, Wahlström J, Fathi M, Rasmussen E, Grunewald J, Tornling G, Lundberg IE: Restricted T cell receptor BV gene usage in the lungs and muscles of patients with idiopathic inflammatory myopathies. Arthritis Rheum 2007, 56(1):372-383.

24. Kurasawa K, Nawata Y, Takabayashi K, Kumano K, Kita Y, Takiguchi Y, Kuriyama T, Sueishi M, Saito Y, Iwamoto I: Activation of pulmonary T cells in corticosteroid-resistant and -sensitive interstitial pneumonitis in dermatomyositis/polymyositis. Clin Exp Immunol 2002, 129(3):541-548.

25. Yamadori I, Fujita J, Kajitani H, Bandoh S, Tokuda M, Ohtsuki Y, Yoshinouchi T, Okahara M, Yamaji Y, Tanimoto $Y$ et al: Lymphocyte subsets in lung tissues of interstitial pneumonia associated with untreated polymyositis/dermatomyositis. Rheumatol Int 2001, 21(3):89-93.

26. Galindo-Feria AS, Albrecht I, Fernandes-Cerqueira C, Notarnicola A, James EA, Herrath J, Dastmalchi M, Sandalova T, Rönnblom L, Jakobsson PJ et al: Proinflammatory Histidyl-Transfer RNA Synthetase-Specific CD4+ T Cells in the Blood and Lungs of Patients With Idiopathic Inflammatory Myopathies. Arthritis Rheumatol 2020, 72(1):179-191.

27. Hozumi H, Fujisawa T, Nakashima R, Johkoh T, Sumikawa H, Murakami A, Enomoto N, Inui N, Nakamura Y, Hosono $Y$ et al: Comprehensive assessment of myositis-specific autoantibodies in polymyositis/dermatomyositis-associated interstitial lung disease. Respir Med 2016, 121:91-99.

28. Gallay L, Gayed C, Hervier B: Antisynthetase syndrome pathogenesis: knowledge and uncertainties. Curr Opin Rheumatol 2018, 30(6):664-673.

29. Guo M, Schimmel P: Essential nontranslational functions of tRNA synthetases. Nat Chem Biol 2013, 9(3):145-153.

30. Casciola-Rosen L, Nagaraju K, Plotz P, Wang K, Levine S, Gabrielson E, Corse A, Rosen A: Enhanced autoantigen expression in regenerating muscle cells in idiopathic inflammatory myopathy. $J$ Exp Med 2005, 201(4):591-601.

31. Feng RN, Niu YC, Sun XW, Li Q, Zhao C, Wang C, Guo FC, Sun CH, Li Y: Histidine supplementation improves insulin resistance through suppressed inflammation in obese women with the metabolic syndrome: a randomised controlled trial. Diabetologia 2013, 56(5):985-994.

32. Friedman AW, Targoff IN, Arnett FC: Interstitial lung disease with autoantibodies against aminoacyltRNA synthetases in the absence of clinically apparent myositis. Semin Arthritis Rheum 1996, 26(1):459-467.

33. Sauty A, Rochat T, Schoch OD, Hamacher J, Kurt AM, Dayer JM, Nicod LP: Pulmonary fibrosis with predominant CD8 lymphocytic alveolitis and anti-Jo-1 antibodies. Eur Respir J 1997, 10(12):29072912.

34. Danoff SK, Casciola-Rosen L: The lung as a possible target for the immune reaction in myositis. Arthritis Res Ther 2011, 13(4):230-230.

\section{Figures}




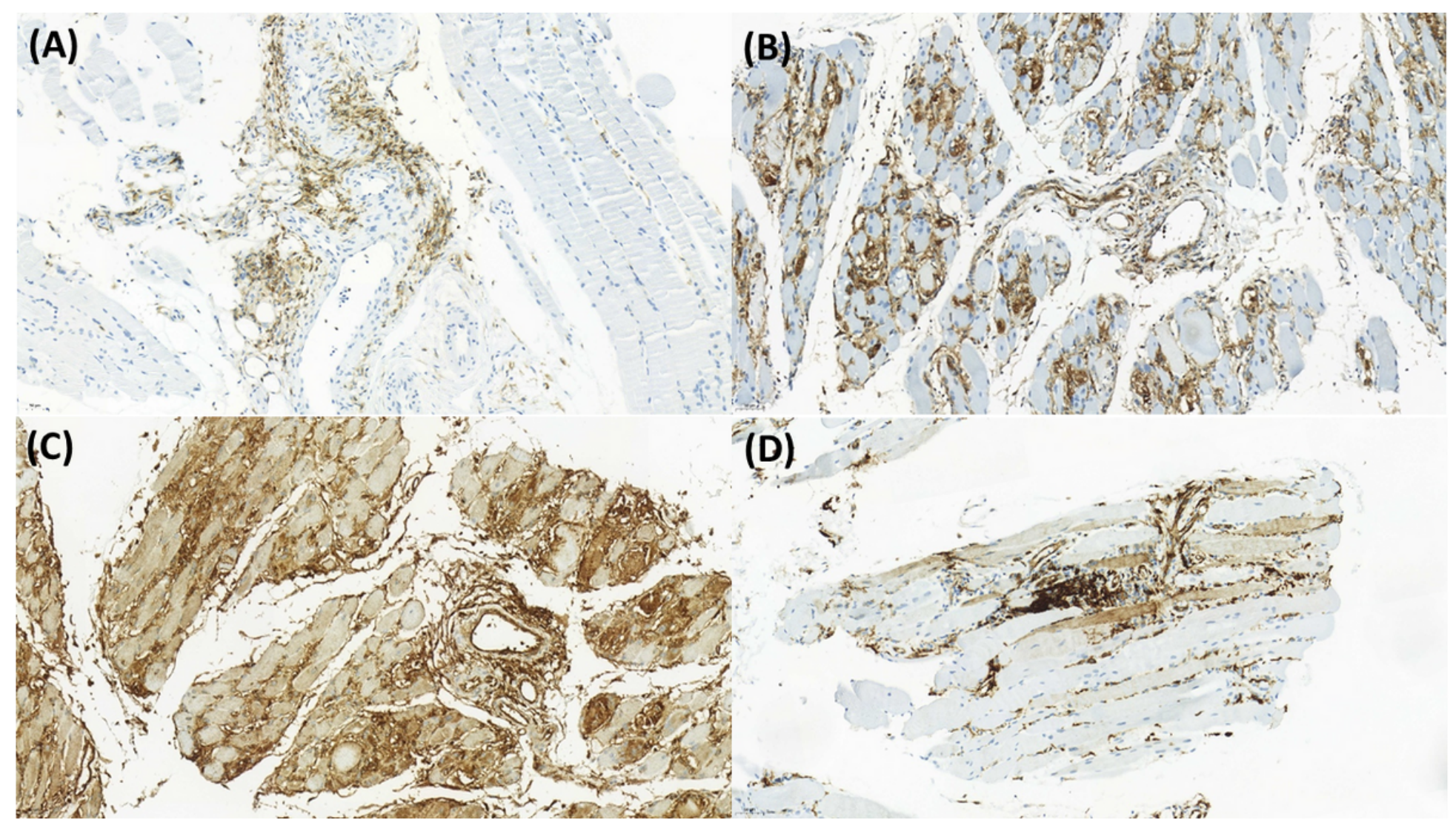

Figure 1

Photomicrographs of CD4, Mx1, MHC class I, and HLA-DR expression in idiopathic inflammatory myopathies. CD4 expression in the endomysial and perivascular areas (A), MX1 expression on immune cells and capillary of dermatomyositis (B), MHC class I expression on immune cells, capillary, and myofibers of dermatomyositis (C), and HLA-DR expression on myofibers (D) are shown. Magnification, $\times 20$. 